\title{
Prediction on Deflection of C-Core Sandwich Panels in Weak Direction
}

\author{
Cui Mingjuan* \\ School of Civil Engineering \& Mechanics, Huazhong University of Science \& Technology, Luoyu Road 1037\#, \\ Hongshan District, Wuhan, Hubei Province, PR China 430074
}

\begin{abstract}
In a C-core sandwich panel, the shear stiffness of the sandwich panel in the weak direction is much smaller. The deflection of a C-core sandwich beam consists of two parts: flexural deformation and shear deformation. The flexural deformation of a C-core sandwich beam can be calculated simply from beam theory while the shear deformation is relatively difficult to calculate due to the mutual action between the facing plates and the C-core stiffener. Considering the contact between the flanges of the C-core and the facing plates, the shear deformation of a typical segment in a C-core sandwich panel is analyzed based on compatibility conditions. By using Castigliano's second theorem, the equation for calculating the deflection caused by shear action of a C-core sandwich panel under bending is obtained. The accuracy of the presented equations for calculating the deflection of a C-core sandwich cantilever beam and a C-core sandwich panel under three-point bending is then verified by comparing with finite element and experimental results reported in the corresponding reference. It is found that the predicted results from the presented equation agree quite well with finite element and experimental results, which shows the reliability and accuracy of the proposed equation.
\end{abstract}

Keywords: C-core sandwich panel, bending behavior, weak direction, shear deflection, compatibility conditions.

\section{INTRODUCTION}

A steel sandwich panel consists of two facing plates and a core between them. This type of structure has a high flexural stiffness, light weight, and especially it has a high resistance to dynamic and blast loads. Due to these advantages, it is widely used in ship and offshore engineering, such as ship and offshore platform decking, or double-skin vessels. There is also a potential application for this structure to be used in building and constructional industries. Combing with light weight materials, such as aluminum, the sandwich panels can be also used in terrestrial and space engineering.

For a steel sandwich panel, the different types are classified according to the cores. Such commonly used types include web-core, C-core, Z-core, X-core, V-core and trusscore etc. The cores are placed generally in a single direction, which causes the sandwich panel to have a different stiffnesses in the two length directions. In the direction of the core placement, the bending stiffness, the shear stiffness and the torsion stiffness are all very high. However, in the direction perpendicular to the core placement, or so-called transverse direction, the shear stiffness is much weaker. The difference of the mechanical properties of a sandwich panel in two directions makes it difficult to analyze this structure in theory because it is essential to be a composite structure. In the literature, much effort has been tried to simplify a sandwich panel to be an equivalent orthotropic thick plate based on providing the appropriate solutions of some fundamental structural properties. Such early representative work was

*Address correspondence to this author at the School of Civil Engineering \& Mechanics, Huazhong University of Science \& Technology, Luoyu Road 1037\#, Hongshan District, Wuhan, Hubei Province, PR China 430074;

Tel: +86-27-87557024;

Email: Cuimingjuan1107@163.com conducted by Libove and Batforf (1948) [1], and Libove and Hubka (1951) [2]. Simplifying a 3D sandwich panel into a $2 \mathrm{D}$ equivalent plate, it is necessary to give accurate solution of some elastic constants such as the bending stiffnesses in two directions, the twisting stiffnesses and the shear stiffnesses in two directions. Libove and Hubka (1951) derived the elastic constants for sandwich panels with a continuous corrugated core based on the assumption that the cross section of the core-stiffeners is symmetrical about a vertical plane. Later on, many researchers spent a lot of effort in deriving accurate solutions of these elastic constants, such as the work reported in Refs. [3-8]. Based on these closed-form solutions of the elastic constants, the static behavior of sandwich panels can be analyzed theoretically and numerically. Cheng et al. (2006) [9] evaluated the accuracy of some elastic constants of sandwich panels with various cores by using finite element analysis. Chang et al. (2005) [10] studied the bending behavior of corrugated-core sandwich panels by using Mindlin-Reissner plate theory. Romanoff et al. $(2006,2007)[11-13]$ analyzed the bending behavior of webcore sandwich beams by using the solutions of elastic constants. Buannic et al. (2003) [14] also investigated the behavior of corrugated core sandwich panels by using a finite element method.

In this paper, C-core sandwich panel under bending in weak direction is studied. The bending behavior of the C-core sandwich beam is then assessed from the load versus deflection relationship. In calculating the beam deflection, a simplified theoretical analysis for deriving the shear deformation is obtained, and a closed form equation for calculating the deflection of the beam due to shear action is presented. The accuracy of this derived equation is then verified using finite element and experimental results. 


\section{THEORETICAL ANALYSIS}

\section{Typical Segment in a C-Core Sandwich Panel}

A C-core sandwich panel consists of two facing plates and a series of C-channel cores, as shown in Fig. (1). The Ccores are connected to the top and bottom facing plates by using laser spot weld or self-tapping screws. The C-cores are placed in parallel only in one direction (along x-axis). In the direction perpendicular to the placement of the $\mathrm{C}$-cores, the sandwich panel has a relatively lower shear stiffness.

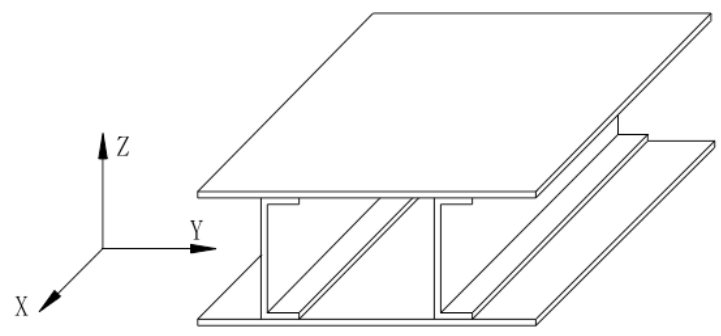

Fig. (1). A C-core sandwich panel.

In case that a C-core sandwich panel is subjected to uniform loading, a typical segment as shown in Fig. (2) can be isolated for analysis. It is assumed that each segment in a Ccore sandwich panel is similar to its adjacent one. This means the deformation of every segment is similar. Therefore, the typical segment can be used to investigate its property, and other segments can be analyzed by using the same method. In Fig. (2), the length of the top and the bottom facing plates in a segment is denoted by $s$. This length is also identical to the distance between any two adjacent $\mathrm{C}$-cores. The distance between the mid-plane of the top and bottom facing plates is denoted by $h_{\mathrm{p}}$. Similarly, $h_{\mathrm{c}}$ is used to represent the distance between the mid-plane of the two flanges of the $\mathrm{C}$-core. The position of the connection between the facing plates and the C-core can be described by $l_{\mathrm{a}}$ and $l_{\mathrm{b}}$. For brevity, the thicknesses of the top and the bottom facing plates are assumed to be same, and such thickness is denoted by $t_{\mathrm{p}}$. The thickness of the C-core, which has a constant value, is denoted by $t_{\mathrm{c}}$.

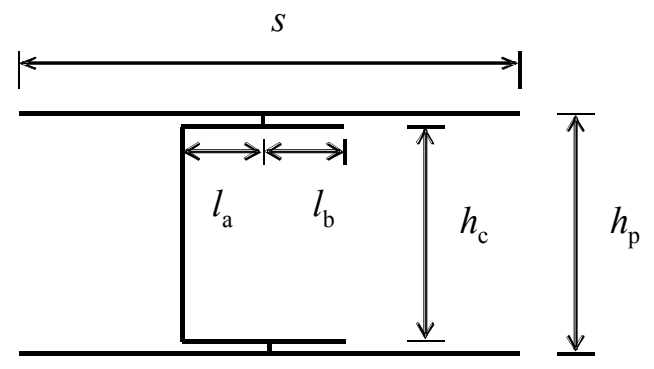

Fig. (2). Typical segment in C-core sandwich panel.

For a C-core sandwich panel under bending, there are no axial forces in a segment while only shear forces and bending moments exist at the left and right cross-sections. As shown in Fig. (3), it is assumed that the bending moments at each end can be equivalently replaced by a couple forces. Hence, the bending moments $M$ at the left end and $M+\Delta M$ at the right end can be replaced by a couple of axial forces in the top and bottom facing plates respectively.

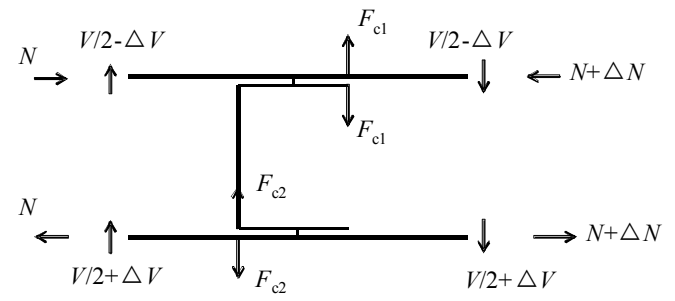

Fig. (3). Equilibrium of segment.

From Fig. (3), the following equations can easily be obtained,

$$
\left\{\begin{array}{l}
M=N h_{p} \\
M+\Delta M=(N+\Delta N) h_{p}
\end{array}\right.
$$

From Eq. (1), it is found that the bending moments at the left and at the right end of the segment is not the same, and the difference is $\Delta M=\Delta N \times h_{p}$. To keep the equilibrium of the segment, the following equation must be satisfied, i.e.

$(V / 2-\Delta V) \times s+(V / 2+\Delta V) \times s=\Delta M$

Eq. (2) can be simplified to the following equation:

$V s=\Delta N h_{p}$ tion

Then, $\Delta N$ can be determined from the following equa-

$\Delta N=V S / h_{p}$

It is noted here that the shear force at the top facing plate is not equal to the shear force at the bottom facing plate in Fig. (3). This assumption is based on the fact that there is a contact force between the facing plates and the flanges of the $\mathrm{C}$-core as shown in Fig. (3). Such contact causes the difference of the shear forces at the top facing plate and at the bottom facing plate. The contact positions are assumed to be located at the end of the C-core flange when shear deformation occurs in the segment, and the corresponding contact forces are denoted by $F_{c 1}$ and $F_{c 2}$ respectively.

It can be seen that there are overall three unknowns $F_{c 1}$, $F_{c 2}$ and $\Delta V$ once the shear force $V$ at both ends of the segment is known since $\Delta N$ can be calculated from Eq. (4). The closed-forms of the three unknowns should be obtained from compatibility conditions.

\section{Compatibility Conditions Between Adjacent Segments}

The compatibility conditions of any typical segment are shown in Fig. (4).

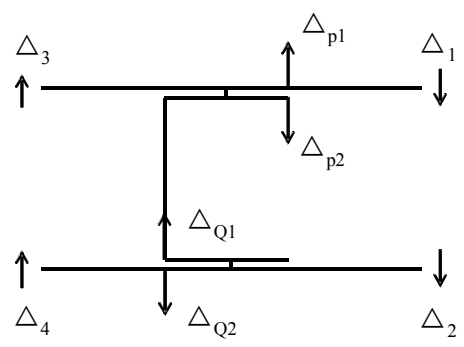

Fig. (4). Compatibility condictions. 
The following two assumptions are made:

(1) There is no relative displacement between the facing plate and the $\mathrm{C}$-core flange at contact point. This assumption is meaningful since there will be no separation between any contact points due to compression action. This assumption will produce the following two equations:

$$
\begin{aligned}
& \Delta_{p 1}+\Delta_{p 2}=0 \\
& \Delta_{Q 1}+\Delta_{Q 2}=0
\end{aligned}
$$

(2) The relative displacement between the top facing plate and the bottom facing plate should be equal at the two ends of the segment. The following equation must be satisfied according to this assumption

$$
\Delta_{2}-\Delta_{1}=\Delta_{3}-\Delta_{4}
$$

Eqs. (5)-(7) can be used to derive the solutions of the three unknowns $F_{c 1}, F_{c 2}$ and $\Delta V$.

\section{Compatibility Equations}

As can be seen from Eqs. (5)-(7), the compatibility conditions are essentially used to calculate the relative displacement at some critical positions. This can be done by using Castigliano's second theorem. To do so, the moment diagram of the segment in equilibrium as shown in (Fig. 3) is drawn first. For brevity, the bending moment $M_{\mathrm{t}}$ can be divided into several parts:

$$
M_{t}=M_{a}(V, \Delta N)+M_{b}(\Delta V)+M_{c}(N)+M_{d}\left(F_{c 1}, F_{c 2}\right)
$$

where $M_{a}(V, \Delta N), M_{b}(\Delta V), M_{c}(N)$ and $M_{d}\left(F_{c 1}, F_{c 2}\right)$ are the moments caused by different loadings.

The moment diagrams produced by $M_{a}(V, \Delta N)$, $M_{b}(\Delta V), M_{c}(N)$ and $M_{d}\left(F_{c 1}, F_{c 2}\right)$ are shown in Figs. (5a-5d) respectively. It should be noted that the segment is always in equilibrium under the external forces as shown in Fig. (5a) to Fig. (5d) respectively.

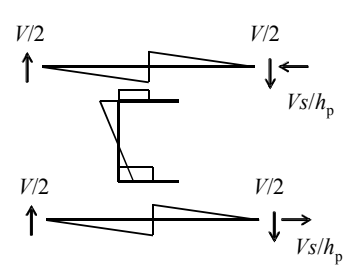

(a) $M_{a}(V, \Delta N)$

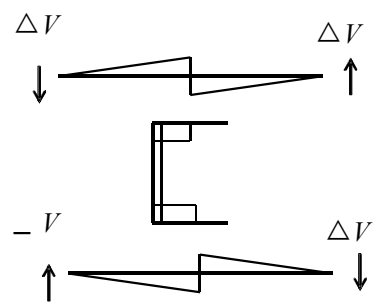

(b) $M_{b}(\Delta V)$
Fig. (5). Moment diagrams of segment.

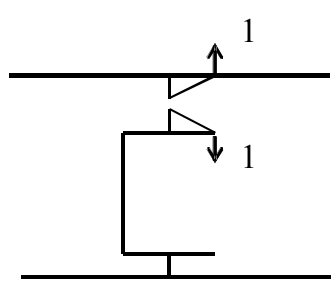

Fig. (6). Moment diagram.
In Eq. (5), to calculate $\Delta_{p 1}+\Delta_{p 2}$, the moment diagram as shown in (Fig. 6) is generated. In Fig. (6), two unit loads are applied at the contact point between the top facing plate and the top C-core flange. Then $\Delta_{p 1}+\Delta_{p 2}$ can be calculated from the following equation

$$
\Delta_{p 1}+\Delta_{p 2}=\int_{\Gamma} \frac{M_{t}}{E I} M_{d}(1,0) d \Gamma
$$

where $M_{d}(1,0)$ means the moment diagram by letting $F_{c 1}=1$ and $F_{c 2}=0 ; E$ is the elastic modulus of the steel material, $I$ is the second moment of area about the mid-plane.

Substituting Eq. (9) into compatibility condition of Eq. (5), the following equation can be obtained,

$$
F_{c 1}=\frac{\beta}{1+\beta} \frac{3}{2 l_{b}}\left[\left(\frac{V}{2}-\Delta V\right)\left(\frac{s}{2}-\frac{l_{b}}{3}\right)\right]
$$

where $\beta=E_{c} I_{c} / E I$ is the ratio of bending stiffness of the $\mathrm{C}$-core stiffener and the facing plates about their respective mid-plane in thickness direction, i.e. for unit width plate along $x$-axis, $I_{c}=t_{c}^{3} / 12$ and $I=t_{p}^{3} / 12$.

Similarly, the compatibility condition at the contact point between the bottom facing plate and the bottom $\mathrm{C}$-core flange can be calculated from the following equation

$$
\Delta_{Q 1}+\Delta_{Q 2}=\int_{\Gamma} \frac{M_{t}}{E I} M_{d}(0,1) d \Gamma
$$

where $M_{d}(0,1)$ is shown in Fig. (7).

From Eq. (6), the following equation can be obtained

$$
F_{c 2}=\frac{\beta}{1+\beta} \frac{3}{2 l_{a}}\left[\left(\frac{s}{2}-\frac{l_{a}}{3}\right)\left(\frac{V}{2}+\Delta V\right)+\frac{s}{\beta}\left(\frac{V h_{c}}{2 h_{p}}+\Delta V\right)\right]
$$

Compatibility condition Eq. (7) can be transformed into the following format

$$
\Delta_{2}-\Delta_{1}-\Delta_{3}+\Delta_{4}=0
$$

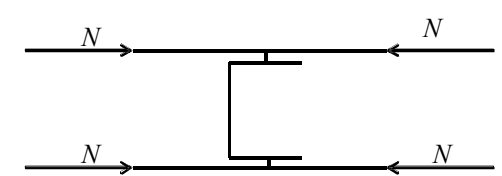

(c) $M_{c}(N)$

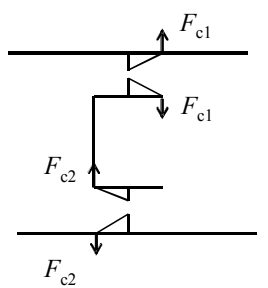

(d) $M_{d}\left(F_{c 1}, F_{c 2}\right)$ 
Similarly, using the moment diagram $M_{b}(1)$ as shown in Fig. (8), the following equation can be obtained as well

$$
\Delta_{2}-\Delta_{1}-\Delta_{3}+\Delta_{4}=\int_{\Gamma} \frac{M_{t}}{E I} M_{b}(1) d \Gamma
$$

Substituting the above equation into Eq. (7), the following equation can be obtained

$$
\Delta V=\frac{\left(-\alpha_{4}+\alpha_{5}+\alpha_{7}\right) \times \frac{V}{2}+\left(\alpha_{5}+\alpha_{6}\right) \frac{V h_{c}}{2 h_{p}}}{\alpha_{1}+\alpha_{2}+\alpha_{3}-\alpha_{4}-2 \times \alpha_{5}-\alpha_{6}-\alpha_{7}}
$$

where

$$
\begin{gathered}
\alpha_{1}=\frac{s^{3}}{6} ; \alpha_{2}=\frac{2 s^{2} l_{a}}{\beta} ; \alpha_{3}=\frac{s^{2} h_{c}}{\beta} ; \alpha_{4}=\frac{3 l_{b}}{4} \frac{\beta}{1+\beta}\left(\frac{s}{2}-\frac{l_{b}}{3}\right)^{2} ; \\
\alpha_{5}=\frac{3 l_{a}}{4} \frac{s}{1+\beta}\left(\frac{s}{2}-\frac{l_{a}}{3}\right) ; \\
\alpha_{6}=\frac{3 l_{a}}{4} \frac{1}{1+\beta} \frac{s^{2}}{\beta} ; \alpha_{7}=\frac{3 l_{a}}{4} \frac{\beta}{1+\beta}\left(\frac{s}{2}-\frac{l_{a}}{3}\right)^{2} \\
1 \\
\downarrow \\
1
\end{gathered}
$$

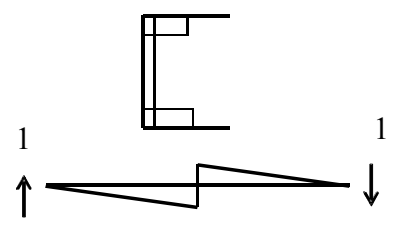

Fig. (8). Moment diagram $M_{b}(1)$.

From Eqs. (10), (12) and (15), it is easy to find that the three unknowns $F_{c 1}, F_{c 2}$ and $\Delta V$ which can be calculated once the value of shear force $V$ at two ends of the segment is known. The steps for calculating the three unknowns are shown in Fig. (9).

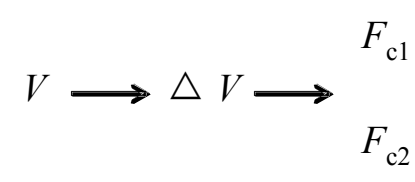

Fig. (9). Steps to calculate the unknowns.

\section{Shear Deformation in Vertical Direction}

After the unknowns are calculated, the shear deformation of the segment shown in Fig. (3) can be analyzed. To calculate a sandwich panel under bending, only the deformation in vertical direction is analyzed, which is shown in Fig. (10). The shear deformation in vertical direction, $\Delta_{\mathrm{s}}$, of this segment is calculated from the following equation:

$$
\Delta_{s}=\frac{\Delta_{1}+\Delta_{2}+\Delta_{3}+\Delta_{4}}{2}
$$

To calculate $\Delta_{\mathrm{s}}$, a moment diagram $\bar{M}$ shown in Fig. (11) is produced. Then $\Delta_{\mathrm{s}}$ is obtained from the following equation:

$$
\Delta_{s}=\frac{1}{2} \int_{\Gamma} \frac{M_{t}}{E I} \bar{M} d \Gamma
$$

Combining Eqs. (10), (12) and (15), the vertical displacement $\Delta_{\mathrm{s}}$ caused by shear action can be calculated from the following equation:

$$
\Delta_{s}=\frac{1}{24 E I}\left[\begin{array}{l}
V s^{3}+\frac{12 l_{a} V s^{2}}{\beta} \frac{h_{c}}{h_{p}}+\frac{2 V s^{2}}{\beta} \frac{h_{c}^{2}}{h_{p}}-6 F_{c 1} l_{b}^{2}\left(\frac{s}{2}-\frac{l_{b}}{3}\right) \\
-\frac{6 F_{c 2} l_{a}^{2} s}{\beta}-6 F_{c 2} l_{a}^{2}\left(\frac{s}{2}-\frac{l_{a}}{3}\right)
\end{array}\right]
$$

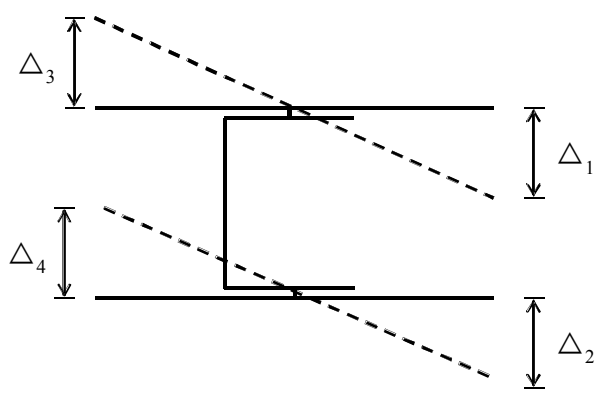

Fig. (10). Shear deformation of a segment.

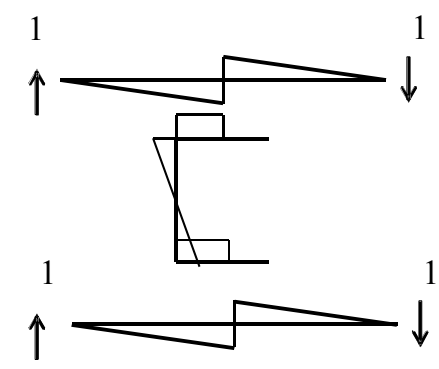

Fig. (11). Moment diagram.

If a sandwich panel consists of $n$ typical segments, then the total vertical displacement of the sandwich panel, $w_{\mathrm{s}}$, is calculated from the following equation:

$$
w_{s}=n \Delta_{s}
$$

As the facing plates and the C-core stiffener in bending behave in a plane strain state, the elastic modulus $E$ in all above equations should be replaced by $E /\left(1-v^{2}\right)$, where $v$ is Poisson's ratio.

\section{Bending Deformation in Vertical Direction}

For a C-core sandwich panel under bending in weak direction, the bending deformation can be calculated simply from the beam theory. However, the second moment of area of the C-core sandwich panel in weak direction, $I_{\mathrm{s}}$, is calculated from the following equation:

$$
I_{s}=\frac{1}{2} t_{p} h_{p}^{2}
$$

Eq. (20) is obtained based on the assumption that the bending moment is sustained by the top and the bottom facing plates while the $\mathrm{C}$-cores do not bear any bending moment. 


\section{CASE STUDY}

To verify the presented method in calculating C-core sandwich panels under bending, two models reported by Fung et al. (1996) are used to assess the accuracy of Eqs. (18) and (19). The two C-core sandwich models include a cantilever beam and a three-point bending panel. The cantilever beam is shown in Fig. (12), and it consists of $11 \mathrm{C}$ channels. The total length of the beam is $800 \mathrm{~mm}$. For the Ccore sandwich panel shown in Fig. (13), the overall $12 \mathrm{C}$ channels are placed between the facing plates. The length and width of the panel are $1000 \mathrm{~mm}$ and $495 \mathrm{~mm}$ respectively. The other detailed dimensions of the cantilever beam and the panel are tabulated in (Table 1). For the cantilever beam, the top and the bottom facing plates are hinged at $\mathrm{A}$ and B, and a line load of $0.125 \mathrm{~N} / \mathrm{mm}$ is applied at the tip ( $V$ $=0.125$ ). For the panel, it is supported at both ends with a span of $1000 \mathrm{~mm}$. The two ends are pinned by just placing them on two supporters. A line load is applied at the midspan of the panel, and the equivalent concentrated load is 5 $\mathrm{kN}(V=5.05 \mathrm{~N} / \mathrm{mm})$.

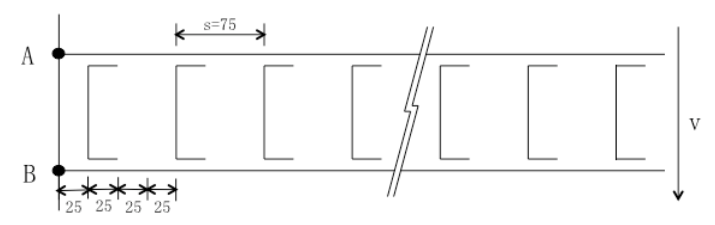

Fig. (12). C-core sandwich cantilever beam.

The overall deflection of the cantilever beam and the panel under bending consists of two parts: the deflection caused by bending $w_{\mathrm{b}}$ and the deflection produced by shear $w_{\mathrm{s}}$. For the cantilever beam, the bending deflection can be simply calculated from beam theory as follow:

$$
w_{b}=\frac{V L^{3}}{3 E I_{s}}
$$

where $L$ is the total length of the cantilever beam, $L=800 \mathrm{~mm}$.
Table 1. Dimensions of a Cantilever Beam and a Panel for CCore Sandwich

\begin{tabular}{|c|c|c|}
\hline Parameter & Cantilever Beam & Panel \\
\hline \hline$s(\mathrm{~mm})$ & 75.0 & 82.2 \\
\hline$l_{\mathrm{a}}(\mathrm{mm})$ & 12.5 & 23.5 \\
\hline$l_{\mathrm{b}}(\mathrm{mm})$ & 12.5 & 13.0 \\
\hline$h_{\mathrm{p}}(\mathrm{mm})$ & 32.4 & 79.0 \\
\hline$h_{\mathrm{c}}(\mathrm{mm})$ & 30.0 & 73.0 \\
\hline$v$ & 0.3 & 0.3 \\
\hline$E\left(\mathrm{~N} / \mathrm{mm}^{2}\right)$ & 68000 & 74400 \\
\hline$E_{\mathrm{c}}\left(\mathrm{N} / \mathrm{mm}^{2}\right)$ & 68000 & 72230 \\
\hline$t_{\mathrm{p}}(\mathrm{mm})$ & 1.2 & 3.0 \\
\hline$t_{\mathrm{c}}(\mathrm{mm})$ & 1.2 & 3.0 \\
\hline
\end{tabular}

For the panel, the bending deflection is calculated from the following equation:

$$
w_{b}=\frac{V L^{3}}{24 E I_{s}}
$$

where $L=1000 \mathrm{~mm}$.

Using Eqs. (19), the shear deflection $w_{\mathrm{s}}$ can also be calculated. For the cantilever beam, the value of $n$ in Eq. (19) is 10.67 , and such value is 5.0 for the panel. The total deflection of the cantilever beam and the panel, $w$, is the sum of $w_{\mathrm{b}}$ and $w_{\mathrm{s}}$.

Fung et al. (1996) provided the finite element result of the cantilever beam and the experimental result of the panel in their research work [4]. For comparison, the predicted results from present work are tabulated together with their results in (Table 2).

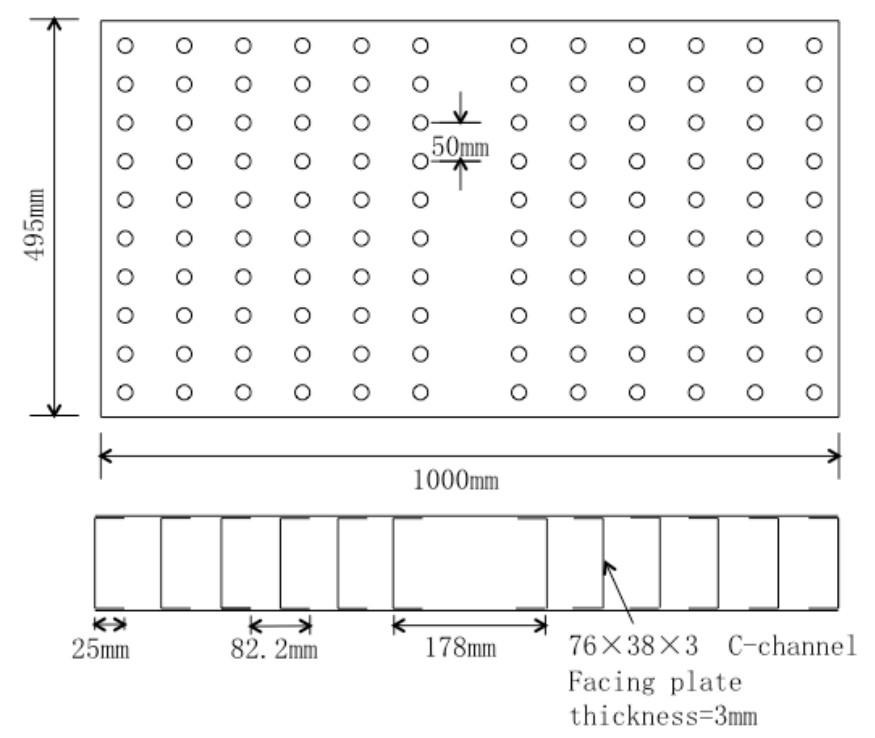

Fig. (13). C-core sandwich panel. 
Table 2. Comparison of the Deflection Between Different Results

\begin{tabular}{|c|c|c|}
\hline Parameter & Beam & Panel \\
\hline \hline Overall deflection by experiment $(\mathrm{mm})$ & - & 13.9 \\
\hline Overall deflection $w$ by FEM $(\mathrm{mm})$ & 6.233 & - \\
\hline Deflection $w_{\mathrm{s}}$ by present method $(\mathrm{mm})$ & 5.822 & 14.36 \\
\hline Deflection $w_{\mathrm{b}}$ by present method $(\mathrm{mm})$ & 0.453 & 0.27 \\
\hline Overall deflection $w$ by present method $(\mathrm{mm})$ & 6.275 & 14.61 \\
\hline Shear force $\Delta V(\mathrm{~N} / \mathrm{mm})$ & 0.0092 & 0.34 \\
\hline Contact force $F_{\mathrm{c} 1}(\mathrm{~N} / \mathrm{mm})$ & 0.107 & 4.585 \\
\hline Contact force $F_{\mathrm{c} 2}(\mathrm{~N} / \mathrm{mm})$ & 0.445 & 10.152 \\
\hline
\end{tabular}

From Table 2, it is found that the deflection of the cantilever beam and the panel calculated from this method shows a good agreement with the finite element or experimental results. A relative error between the results calculated from the present method and the results reported by Fung et al. (1996) is defined as follow:

$$
e=\frac{w_{1}-w_{0}}{w_{0}} \times 100 \%
$$

Where $w_{1}$ is the deflection calculated from the presented equations in this study, $w_{0}$ is the results reported by Fung et al. (1996).

For the cantilever beam and the panel, the values of the relative error are $e=0.67 \%$ and $e=5.0 \%$ respectively. The small errors show a good agreement between the theoretical results and the finite element or experimental results. This means the presented method in this study is reliable and accurate for predicting the bending behaviour of $\mathrm{C}$-core sandwich panels.

Additionally, it can also be found from the results in (Table 2) that deflection due to shear is very dominant in the overall deflection for both the cantilever beam and for the panel. The deflection caused by bending contributes only a small percentage. Thus, it is important to consider the shear deformation for a Core-sandwich panel under bending in its weak direction.

\section{CONCLUSIONS}

The bending behavior of C-core sandwich panel is investigated in the present study, and an equation for predicting the shear deformation of C-core sandwich panel in the weak direction is derived. Through case study, the following two conclusions can be made:

1). The presented equation in this study is accurate and reliable in calculating the deflection of C-core sandwich panel under bending.

2). For C-core sandwich panel under bending in weak direction, the shear deformation is much bigger than the flexural deformation.

\section{CONFLICT OF INTEREST}

The authors confirm that this article content has no conflicts of interest.

\section{ACKNOWLEDGEMENT}

Declared none.

\section{REFERENCES}

[1] C. Libove and S.B. Batdorf, A general small deflection theory for flat sandwich plates, NACA Tech. Note 1526, Nat. Advisory Com. for Aeronautics, Washingtong D.C., 1948.

[2] C. Libove and R.E. Hubka, Elastic constants for corrugated-core sandwich plates, NACA Tech. Note 2289, Nat. Advisory Com. for Aeronautics, Washington, D.C., 1951.

[3] T. Nordstrand, L.A. Carlsson and H.G. Allen, "Transverse shear stiffness of structural core sandwich," Composite Struct., vol. 27, pp. 317-329, 1994.

[4] T.C. Fung, K.H. Tan and T.S. Lok, "Shear stiffness $D_{\mathrm{Qy}}$ for C-core sandwich panels," J. Struc. Eng., ASCE, vol. 122, pp. 958-966, 1996.

[5] T.C. Fung and K.H. Tan, "Shear stiffness for Z-core sandwich panels,” J. Struct. Eng., ASCE, vol. 124, pp. 809-816, 1998.

[6] T.C. Fung, K.H. Tan and T.S. Lok, "Elastic constants for Z-core sandwich panels," J. Struct. Eng., ASCE, vol. 120, pp. 3046-3055, 1994.

[7] T.S. Lok, Q.H. Cheng, Elastic stiffness properties and behaviour of truss-core sandwich panel," J. Struct. Eng., ASCE, vol. 126, pp. 552-559, 2000.

[8] P. Kujala, A. Klanac, Analytical and numerical analysis of nonsymmetrical all steel sandwich panels under uniform pressure load. In international design conference - design 2002, Dubrovnik, 2002, 1205-1210.

[9] Y.B. Shao, S.T. Lie and S.P. Chiew, "Static strength of tubular Tjoints with reinforced chord under axial compression," Advances Struct. Eng., vol. 13, pp. 369-378, 2010.

[10] W.S. Chang, E. Ventsel, T. Krauthammer and J. John, "Bending behavior of corrugated-core sandwich plates," Composite Struct., vol. 70, pp. 81-89, 2005.

[11] J. Romanoff and P. Varsta, "Bending response of web-core sandwich beams," Composite Struct., vol. 73, PP. 478-487, 2006.

[12] J. Romanoff and P. Varsta, "Bending response of web-core sandwich plates," Composite Struct., vol. 81, pp. 292-302, 2007.

[13] J. Romanoff, P. Varsta and A. Klanac, "Stress analysis of homogenized web-core sandwich beams," Composite Struct., vol. 79, pp. 411-422, 2007.

[14] N. Buannic, P. Cartraud and T. Quesnel, "Homogenization of corrugated core sandwich panels," Composite Struct., vol. 59, pp. 299$312,2003$. 